\title{
Sentinel lymph node mapping in endometrial cancer - areas where further research is needed
}

\author{
Andreas Obermair, ${ }^{1}$ Nadeem R Abu-Rustum ${ }^{2}$
}

\begin{abstract}
${ }^{1}$ Queensland Centre for Gynaecological Cancer, Herston/ Brisbane, Queensland, Australia ${ }^{2}$ Surgery, Memorial SloanKettering Cancer Center, New York City, New York, USA
\end{abstract}

\section{Correspondence to}

Professor Andreas Obermair, Queensland Centre for Gynaecological Cancer, Herston/ Brisbane, QLD 4029, Australia; ao@surgicalperformance.com

Received 17 November 2019 Accepted 19 November 2019 Published Online First 17 January 2020

Check for updates

(C) IGCS and ESG0 2020. № commercial re-use. See rights and permissions. Published by BMJ.

To cite: Obermair A, Abu-Rustum NR. Int J Gynecol Cancer 2020;30:283-284.
Over the past decades the surgical treatment of endometrial cancer has undergone major changes, including a shift away from open toward minimally invasive surgery. Current standard treatment now involves minimally invasive hysterectomy and bilateral salpingo-0ophorectomy with or without surgical staging. Before the concept of surgical staging developed, the treatment of endometrial cancer consisted of hysterectomy with or without radiation treatment depending on uterine risk factors. In 1987, the GOG33 $^{1}$ study suggested that a sizeable number of patients with clinical stage 1 endometrial cancer had disease outside the uterus (lymph nodes, adnexa, peritoneum). This study described uterine pathologic factors that increased the risk of pelvic and aortic lymph node involvement, including higher tumor grade and depth of myometrial invasion.

In 1988, FIG0 transitioned the existing clinical staging to a surgical staging system for endometrial cancer, which includes histological assessment of uterus, cervix, tubes and ovaries, pelvic cytology, and lymph nodes. The adoption of pelvic and aortic lymphadenectomy for staging was not based on randomized controlled trials or evidence demonstrating improved survival. Since then, the issue of whether all patients require a lymph node dissection and the extent of the node dissection has been one of the most controversial issues in gynecological oncology. In 2008 and 2009, two randomized trials did not show a survival benefit in patients who underwent a lymph node dissection, compared with patients who did not have a node dissection. ${ }^{23}$ However, a greater proportion of women in the node dissection groups developed surgical adverse events, including lymphedema. Both trials were reviewed critically because aortic node dissection was not mandated, most likely because it would have been technically most challenging, infeasible, and unsafe specifically in obese patients. It was also argued that the requirements for postoperative treatment (chemotherapy, radiation treatment, chemoradiation) were poorly specified in both trial protocols.

Subsequently, gynecological oncologists used a large variety of surgical staging strategies ranging from full bilateral pelvic +/- aortic node dissection; selected node dissection based on intraoperative frozen section results; random node sampling; or no-node dissection. ${ }^{4}$ Sentinel lymph node mapping evolved as an alternative concept to a full lymph node dissection. According to the sentinel lymph node concept, tumor cells metastasize to one or a few lymph nodes before involving further lymph nodes. A tracer injected interstitially in the vicinity of the tumor, is transported via local lymphatic channels toward the draining lymphatic basins, and the first node that the tracer reaches is called the "sentinel node". The sentinel lymph node mapping technique involves a process that allows the selective removal of few lymph nodes. Algorithms to facilitate the new adoption of the sentinel lymph node mapping technique were created to facilitate learning and were based on single institution experiences. A negative sentinel node should indicate that secondary echelon nodes are truly negative and not involved, making a removal of these nodes unnecessary.

The FIRES trial $\left.\right|^{5}$ was a prospective, non-randomized clinical trial of sentinel lymph node mapping followed by full pelvic $+/-$ aortic node dissection. Of 340 patients who had sentinel lymph node biopsy, 41 (12\%) patients had positive nodes. The authors reported a sensitivity to detect node-positive disease of $97.2 \%$ and a negative predictive value of $99.6 \%$, concluding that sentinel lymph node mapping has a high diagnostic accuracy and can safely replace lymphadenectomy in the staging of endometrial cancer. ${ }^{5}$

Near-infrared technology and the use of indocyanine green (ICG) recently emerged as the dye of choice for sentinel lymph node mapping. A noninferiority, randomized trial of 180 patients with uterine or cervical cancer found that, in total, $471(97 \%)$ of 485 lymph nodes were identified with ICG and 226 (47\%) with isosulfan blue. ${ }^{6}$ It was established that ICG injected into the cervix should be considered the new standard of care for sentinel lymph node mapping in women with cervical and uterine cancers.

At the time of writing this editorial, the majority of gynecological oncologists agree that sentinel lymph node mapping should be part of standard surgical management. A recent survey among 489 


\section{Editorial}

gynecological oncologists from Europe and the USA confirmed that sentinel lymph node mapping was already used by $50.3 \%$ and $69.5 \%$ of gynecological oncologists in Europe and the USA, respectively. $^{7}$

However, three important questions remain unanswered:

1. Sentinel lymph node mapping technique: With the rapid and global adoption of sentinel lymph node mapping comes the variability of surgical technique. Research is required to offer consensus on the mandatory, optional, and unwarranted steps of sentinel lymph node mapping that will lead to a harmonization of the surgical technique and allow appropriate training and assessment of optimal surgical performance to be performed globally.

2. Ultrastaging: Ultrastaging involves additional sectioning and staining of the sentinel lymph node with H\&E and immunohistochemistry to examine the node for low-volume metastatic disease. Optimal histopathological and/or molecular assessment protocols of nodal tissue remains unknown and important questions remain concerning the clinical management of patients with (very) low-volume disease.

3. Patient outcomes: It remains unknown how sentinel lymph node mapping will impact patient outcomes, including the need for postoperative radiation treatment or chemotherapy, quality of life, incidence of adverse events, and survival. The lack of highlevel evidence on the impact of sentinel lymph node mapping on these patient outcomes needs to be addressed as an urgent research priority.

In the past, the establishment of surgical techniques, including a transition from open to minimally-invasive surgery in gynecological cancer, sometimes evolved despite the lack of high-level clinical evidence on its effectiveness and patient outcomes. Creating evidence in these areas will assist gynecological oncologists, pathologists, and medical and radiation oncologists in clinical decision-making concerning endometrial cancer management. It will also assist patients to make informed choices about treatment options.
The present statement urges clinicians and researchers to consider the generation of new knowledge in these three areas. High-level evidence generated through robust study designs may assure clinicians and patients, lead to changes in surgical technique, improved outcomes for women diagnosed with gynecological cancer, and, finally, to clinical practice change.

Funding The authors have not declared a specific grant for this research from any funding agency in the public, commercial, or not-for-profit sectors.

Editor's note This editorial will feature in a special issue on sentinel lymph node mapping in 2020.

Competing interests None declared.

Patient consent for publication Not required.

Provenance and peer review Not commissioned; externally peer reviewed.

\section{REFERENCES}

1 Creasman WT, Morrow CP, Bundy BN, et al. Surgical pathologic spread patterns of endometrial cancer: a gynecologic Oncology Group study. Cancer 1987;60:2035-41.

2 Benedetti Panici P, Basile S, Maneschi F, et al. Systematic pelvic lymphadenectomy vs. no lymphadenectomy in early-stage endometrial carcinoma: randomized clinical trial. J Natl Cancer Inst 2008;100:1707-16.

3 Kitchener H, Swart AMC, Qian Q, et al. Efficacy of systematic pelvic lymphadenectomy in endometrial cancer (MRC ASTEC trial): a randomised study. Lancet 2009;373:125-36.

4 Fotopoulou C, Kraetschell R, Dowdy S, et al. Surgical and systemic management of endometrial cancer: an international survey. Arch Gynecol Obstet 2015;291:897-905

5 Rossi EC, Kowalski LD, Scalici J, et al. A comparison of sentinel lymph node biopsy to lymphadenectomy for endometrial cancer staging (FIRES trial): a multicentre, prospective, cohort study. Lancet Oncol 2017;18:384-92.

6 Frumovitz M, Plante M, Lee PS, et al. Near-infrared fluorescence for detection of sentinel lymph nodes in women with cervical and uterine cancers (FILM): a randomised, phase 3, multicentre, non-inferiority trial. Lancet Oncol 2018;19:1394-403.

7 Casarin J, Multinu F, Abu-Rustum N, et al. Factors influencing the adoption of the sentinel lymph node technique for endometrial cancer staging: an international survey of gynecologic oncologists. Int J Gynecol Cancer 2019;29:60-7. 